\title{
Controlled Synthesis of 2-D and 3-D Dendritic
}

\section{Platinum Nanostructures}

Yujiang Song, ${ }^{* \dagger}$ Yi Yang, ${ }^{*}$ Craig J. Medforth, ${ }^{*}$ Eulalia Pereira, ${ }^{*}$ Anup K. Singh, ${ }^{*}$ Huifang Xu, Yingbing Jiang, ${ }^{\dagger}$ C. Jeffrey Brinker, ${ }^{* \dagger}$ Frank van Swol, ${ }^{* \dagger}$ and John A. Shelnutt ${ }^{* \S}$

*Advanced Materials Laboratory, Sandia National Laboratories, Albuquerque, NM 87106

${ }^{\dagger}$ Departments of Chemistry, Chemical and Nuclear Engineering, and Earth and Planetary Sciences, University of New Mexico, Albuquerque, NM 87131

${ }^{\ddagger}$ CEQUP/Departamento de Quimica, Faculdade de Ciencias, Universidade do Porto, Porto, Portugal

${ }^{\S}$ Department of Chemistry, University of Georgia, Athens, GA 30602

E-mail Address: jasheln@unm.edu 


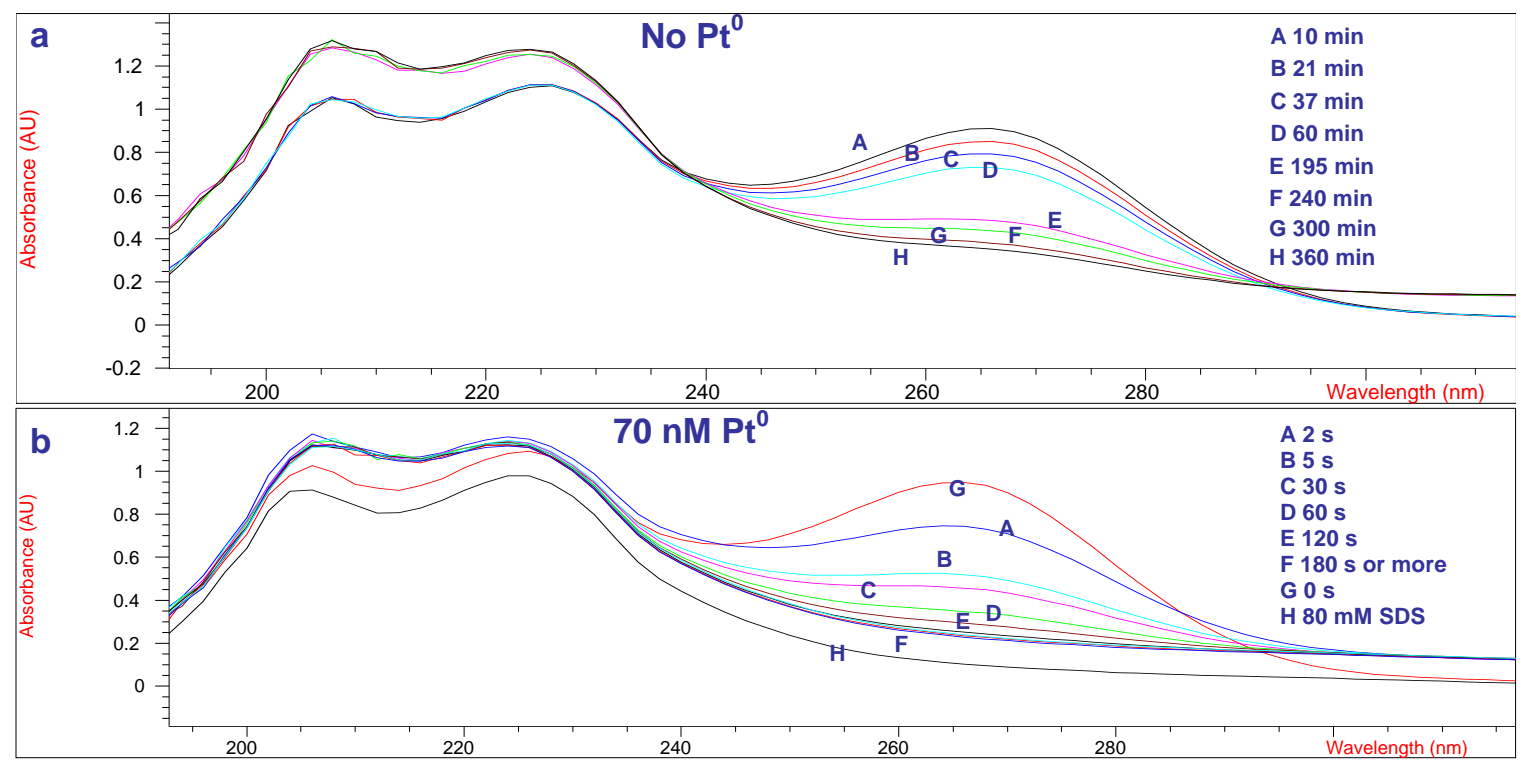

Figure S1. (a) UV spectra of ascorbic acid (7.5 mM in $80 \mathrm{mM}$ SDS) showing gradual oxidation with time in air in the absence of $\mathrm{Pt}^{0}$ and (b) rapid oxidation in air in the presence of $70 \mathrm{nM} \mathrm{Pt}^{0}$. 

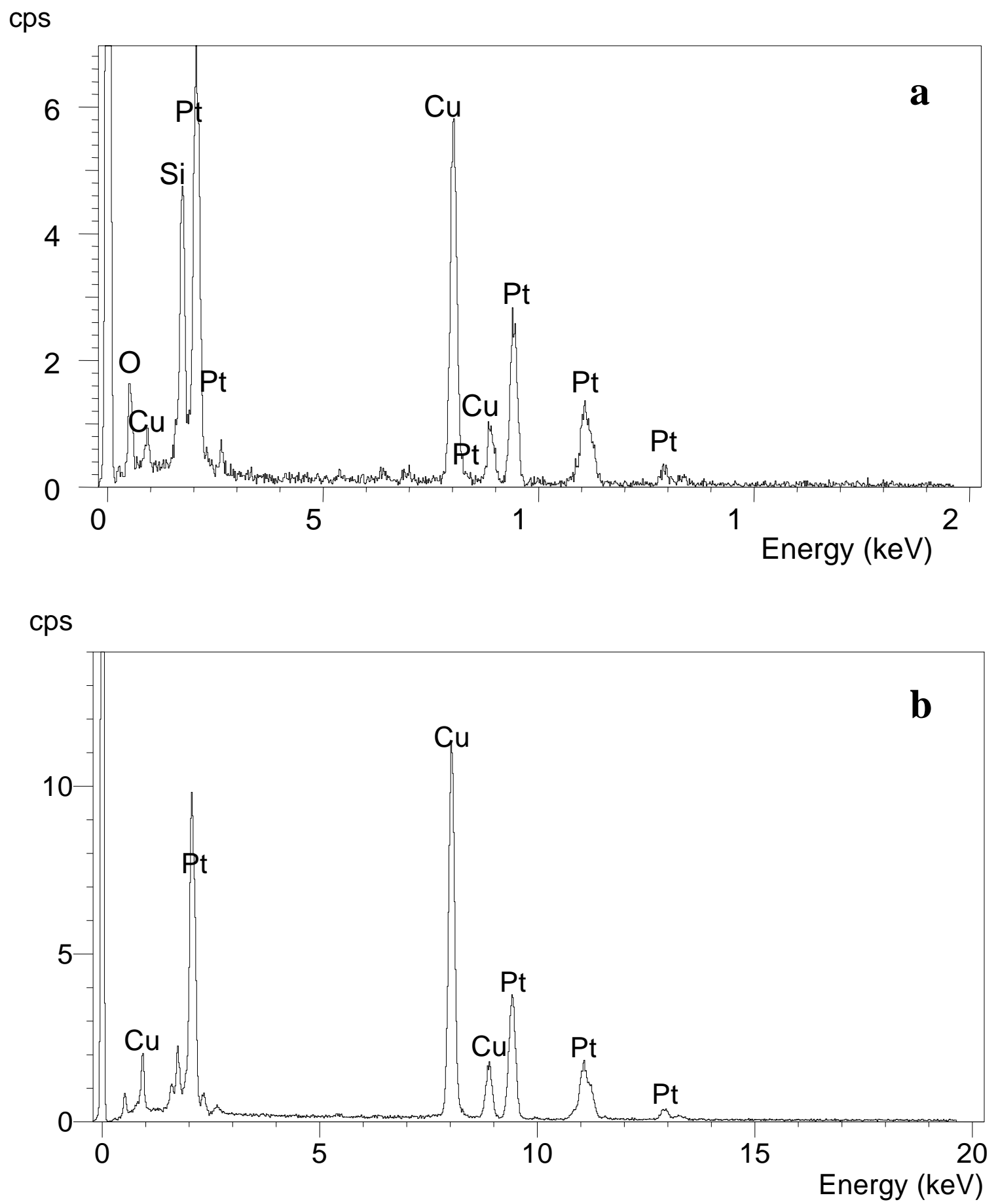

Figure S2. (a) Energy-dispersive X-ray spectroscopy of nanocaps. (b) Energy-dispersive X-ray spectroscopy of 3-D nanodendrites. The $\mathrm{Cu}$ is from the copper TEM grid. 

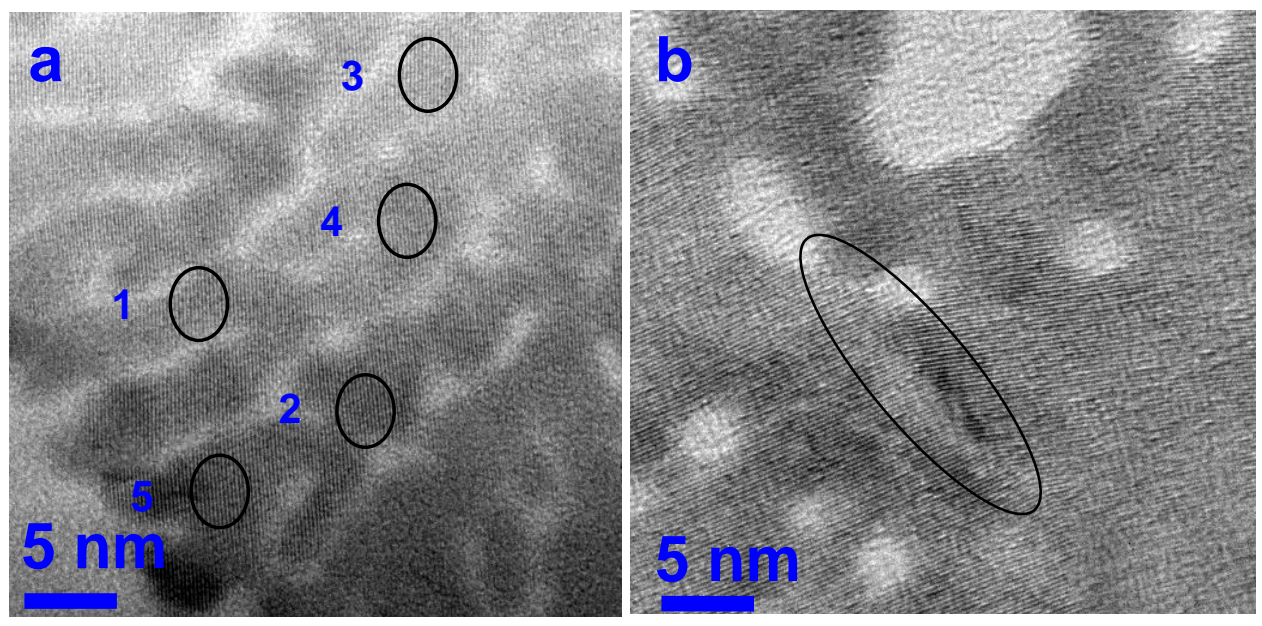

Figure S3. (a) High resolution TEM image of a nanocap showing five circled areas with different fringing angles; the angles are $87.3^{\circ}(1), 84.3^{\circ}(2), 89.5^{\circ}(3), 83.6^{\circ}(4)$, and $85.4^{\circ}(5)$, respectively; (b) image showing a circled area with a low-angle grain boundary. 


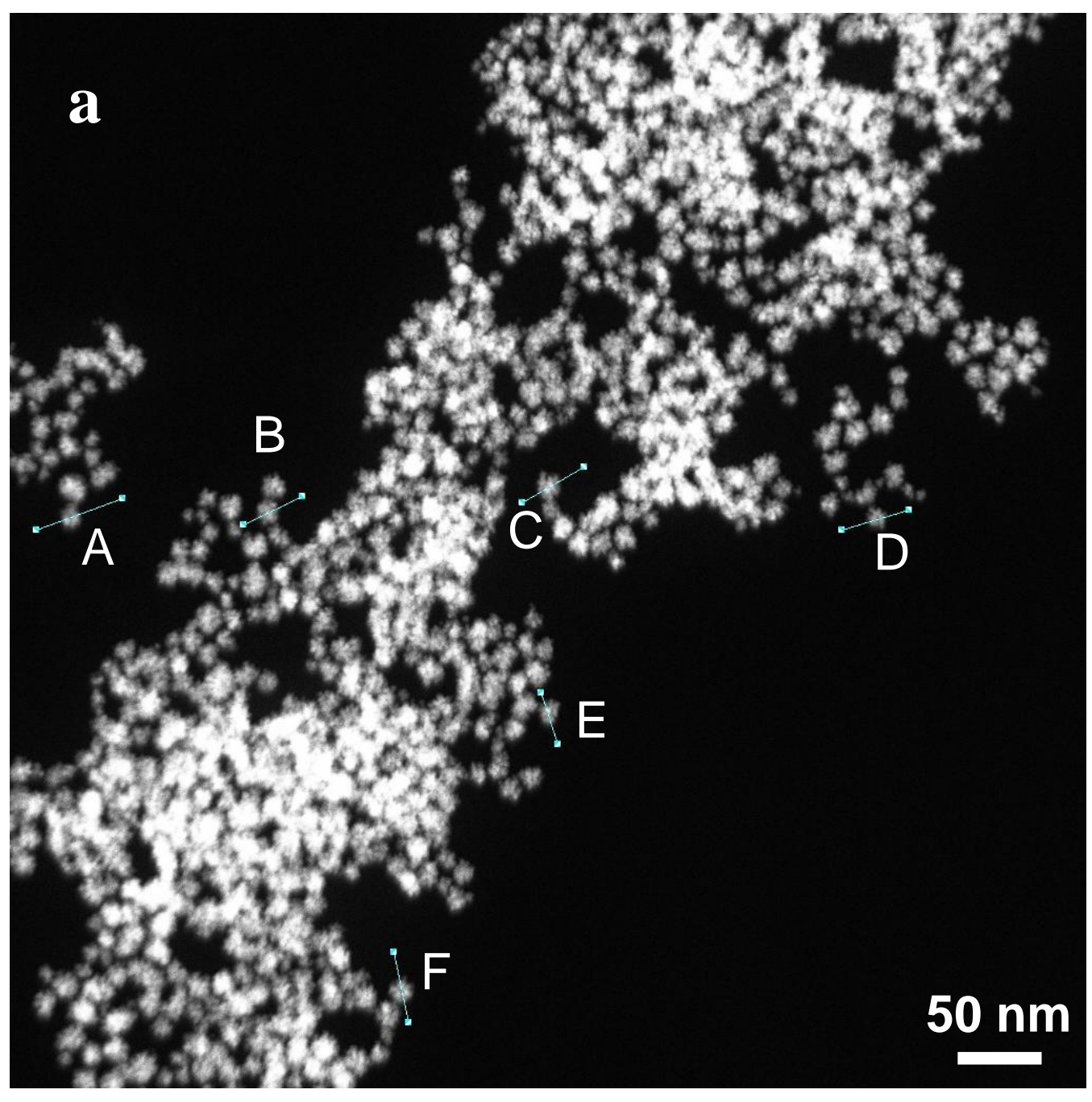



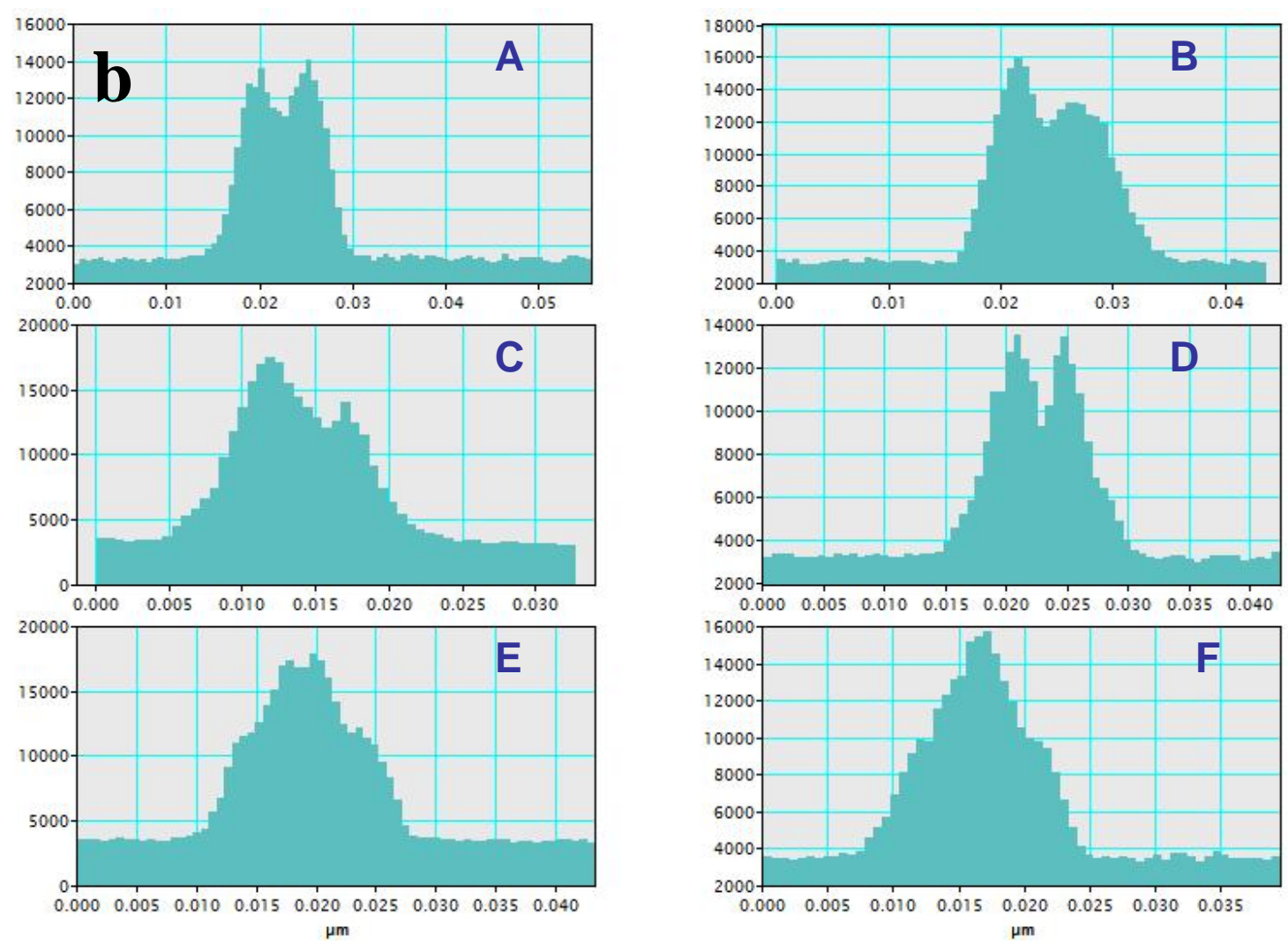

Figure S4. (a) HAADF scanning TEM image of 3-D nanodendrites and (b) density profiles for six randomly selected nanodendrites in (a). 